\title{
3D EVALUATION OF THE KNEE JOINT FUNCTIONING USING AN AMBULATORY SYSTEM: APPLICATION TO ACL-DEFICIENT KNEES
}

\author{
J Favre $^{1}$, B Jolles ${ }^{2}$, F Luthi $^{2}$ and K Aminian ${ }^{1}$ \\ ${ }^{1}$ Ecole Polytechnique Fédérale de Lausanne (EPFL), Laboratory of Movement Analysis and Measurement (LMAM) \\ ${ }^{2}$ University Hospital of Lausanne (CHUV), Orthopaedic Hospital (HOSR) \\ Lausanne, Switzerland \\ email: julien.favre@epfl.ch
}

\section{INTRODUCTION}

Anterior Cruciate Ligament (ACL) injuries may lead to knee joint instability, which in turn may cause meniscal tears and cartilage lesions. Although several studies have shown that injuries of the ACL affect performances of daily activities, the three dimensional (3D) gait patterns after ACL injury and/or surgery are not yet clearly understood.

The purpose of this study was twofold: firstly, to develop an ambulatory system based on inertial sensors which enables the evaluation of 3D knee kinematics, and secondly, to compare the differences between ACL-deficient and healthy knees, before and after operation.

\section{METHODS}

Four young patients with a median age of 24 years (range 17-30) with symptomatic isolated ACL deficiency participated in this study. Patients were evaluated a first time after injury and complete knee function recovery (E1) and a second time, five months (range 4-7) after surgery (E2). Each evaluation consisted in laxity measurement using a $\mathrm{KT}-1000^{\mathrm{TM}}$ arthrometer, classification according to the IKDC score, and $30 \mathrm{~m}$ of walk at comfortable pace monitored by the proposed system.

The movement analysis device was composed of two small sensory units, each including a 3D gyroscope and a 3D accelerometer, fixed by straps on the thigh and shank of the patients. Signals were sampled (@240 Hz) and recorded by an ambulatory datalogger (Physilog ${ }^{\circledR}$ ). The orientations of the two units during the movement were calculated according to the algorithm proposed by Favre et al. [1]. The location and orientation of each unit relative to the body segments were corrected by a calibration procedure described in Favre et al. [2]. This procedure allows a repeatable measurement of the $3 \mathrm{D}$ knee rotation (flexion/extension F/E, abduction/adduction $\mathrm{A} / \mathrm{A}$ and internal/external rotation $\mathrm{I} / \mathrm{E}$ ) as recommended by the ISB [3]. The gait cycles were determined according to Aminian et al. [4] and the mean cycle $3 \mathrm{D}$ joint rotations $(\overline{F / E}$, $\overline{A / A}, \overline{I / E}$ ) were calculated on the steady part of the walk. The functioning agreement $\left(\alpha_{F E}, \alpha_{A A}, \alpha_{I E}\right)$ between the ACL-deficient and healthy knee was calculated for the three angles as follow (similar relations exist for $\alpha_{A A}, \alpha_{I E}$ ):

$\alpha_{F / E}=\sum_{i=0}^{100 \% \text { cycle }}\left|\overline{F / E}_{\text {Healthy }}(i)-\overline{F / E}_{\text {ACL-deficient }}(i)\right|$, deg

The changes in the functioning agreement between both evaluations (E1 and E2) were evaluated for each angle by the $\beta$ parameter, which represents the improvement of the functioning agreement from the first to the second evaluation.

$\beta_{F / E}=\left[a_{F / E}(E 1)-a_{F / E}(E 2)\right] / a_{F / E}(E 1) * 100, \quad \%$

\section{RESULTS AND DISCUSSION}

Both the IKDC score (E1: 2C + 2D; E2: $1 \mathrm{~A}+3 \mathrm{~B})$ and the laxity measurement (side to side differences: E1: 6-10 mm; E2: $1-4 \mathrm{~mm}$ ) reported an improvement for all the patients at E2. Figure 1 displays the three mean cycle rotation of the ACL-deficient and healthy knee of a typical subject at evaluation E1 and E2 and Table 1 reports the $\beta$ parameters for the four subjects.

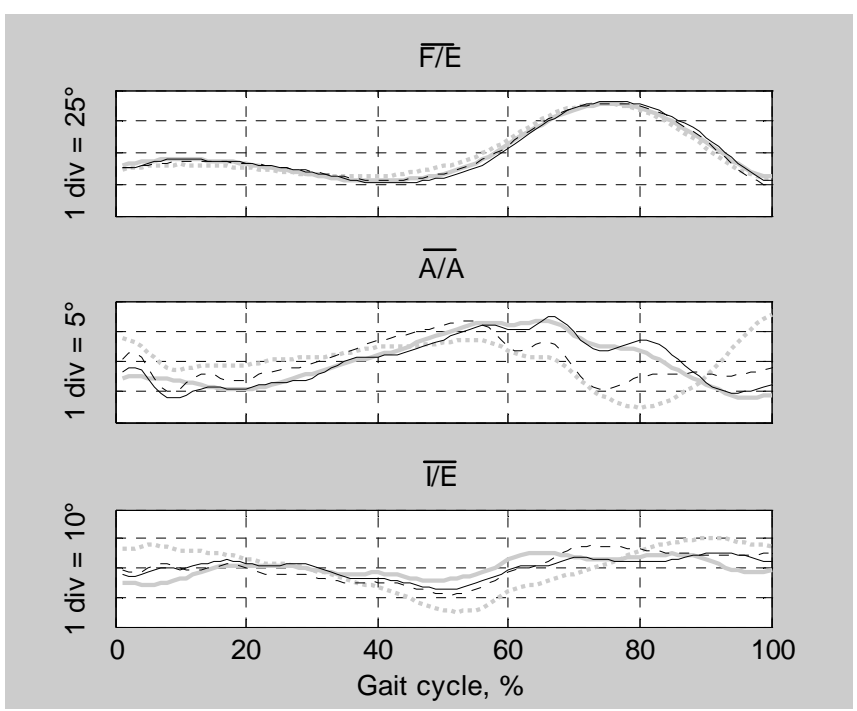

Figure 1: Mean cycle ACL-deficient knee angles are displayed in dotted line and healthy knee in full line. Values in gray correspond to $\mathrm{E} 1$ and values in black to E2.

Table 1: Improvement estimated by the $\beta$ parameters

\begin{tabular}{|c|c|c|c|c|}
\hline & Patient 1 & Patient 2 & Patient 3 & Patient 4 \\
\hline$\beta_{\mathrm{F} / \mathrm{E}}, \%$ & 41 & -34 & 3 & 78 \\
\hline$\beta_{\mathrm{A} / \mathrm{A}}, \%$ & 38 & 75 & 26 & -31 \\
\hline$\beta_{\mathrm{IIE}}, \%$ & 69 & 7 & 47 & 56 \\
\hline
\end{tabular}

The 3D mean cycle angles measured by the proposed system (Figure 1) were in agreement with the values reported in the literature. Moreover, these primary clinical results (Table 1) showed a general improvement in the 3D knee functioning agreement after surgery which is in accordance with the IKDC score and the laxity measurement.

This study presented a new ambulatory system for the estimation of the 3D knee joint angles using inertial sensors. Based on this system it was possible to perform a gait analysis on a long distance and to evaluate the agreement between the functions of the healthy and ACL-deficient knee. Although the results of this study have been interpreted with caution given the small number of patients, they were very encouraging for future evaluation of joint function.

\section{REFERENCES}

1. Favre J, et al. Electronics Letters 42, 612-614, 2006.

2. Favre J, et al. Proceedings of ISB-3D, France, Abstract 112, 2006.

3. Grood ES, et al. J Biom Eng 105, 136-144, 1983.

4. Aminian K, et al.. J Biomech 35, 689-699, 2002 